\title{
Using a Grandfather Pendulum Clock to Measure the World's Shortest Time Interval, the Planck Time (With Zero Knowledge of $G$ )
}

\author{
Espen Gaarder Haug \\ Norwegian University of Life Sciences, Ås, Norway \\ Email: espenhaug@mac.com
}

How to cite this paper: Haug, E.G. (2021) Using a Grandfather Pendulum Clock to Measure the World's Shortest Time Interval, the Planck Time (With Zero Knowledge of $G$ ). Journal of Applied Mathematics and Physics, 9, 1076-1088.

https://doi.org/10.4236/jamp.2021.95074

Received: April 24, 2021

Accepted: May 24, 2021

Published: May 27, 2021

Copyright $\odot 2021$ by author(s) and Scientific Research Publishing Inc. This work is licensed under the Creative Commons Attribution International License (CC BY 4.0).

http://creativecommons.org/licenses/by/4.0/

\begin{abstract}
Haug has recently introduced a new theory of unified quantum gravity coined "Collision Space-Time". From this new and deeper understanding of mass, we can also understand how a grandfather pendulum clock can be used to measure the world's shortest time interval, namely the Planck time, indirectly, without any knowledge of $G$. Therefore, such a clock can also be used to measure the diameter of an indivisible particle indirectly. Further, such a clock can easily measure the Schwarzschild radius of the gravity object and what we will call "Schwarzschild time". These facts basically prove that the Newton gravitational constant is not needed to find the Planck length or the Planck time; it is also not needed to find the Schwarzschild radius. Unfortunately, there is significant inertia towards new ideas that could significantly alter our perspective on the fundamentals in the current physics establishment. However, this situation is not new in the history of science. Still, the idea that the Planck time can be measured totally independently of any knowledge of Newton's gravitational constant could be very important for moving forward in physics. Interestingly, an old instrument that today is often thought of as primitive instrument can measure the world's shortest possible time interval. No atomic clock or optical clock is even close to be able to do this.
\end{abstract}

\section{Keywords}

Pendulum Clock, Planck Time, Planck Length, Planck Scale, Planck Constant, Schwarzschild Radius, Schwarzschild Time, Collision Time, Newton's

Gravitational Constant, Huygens

\section{Important Elements in the History of Gravity}

Before we show how to measure the Planck time with a grandfather clock, we 
will briefly summarize the key points in the history of gravity that are relevant to this paper.

- 1656-Pendulum clock invented by Christiaan Huygens.

- 1673-Christiaan Huygens [1] publishes theory on how to calculate the pendulum periodicity from gravity acceleration: $T=2 \pi \sqrt{\frac{L}{g}}$.

- 1687, 1713, and 1726-Three versions of the Principia. Newton's [2] gravitational formula $F=\frac{M m}{r^{2}}$. Newton only states this formula in words. Newton never introduced a gravitational constant per se, nor did he have any use for one. Newton stated that mass is proportional to weight. Further, he showed how to extract the relative mass of astronomical objects easily. Based on the size of the planets, he could find their relative densities. Newton was focusing on relative masses, as also pointed out by Cohen [3]; "That is, since Newton is concerned with relative masses and densities, the test mass can take any unity, so that weight-force may be considered the gravity or gravitational force per unit mass...".

- 1796-The introduction of the kilogram (kg), but the kilogram gets widely accepted first in Europe after the Metre Convention was signed in 1875, which again leads to the production of The International Prototype of the Kilogram.

- 1798-Cavendish [4] calculates the density of the Earth relative to known uniform elements such as water, lead, or gold using a torsion balance. This torsion balance was invented by Cavendish's friend John Michell sometime before 1783, but Michell was not able to perform the experiment before he died. However, Cavendish never mentions a gravitational constant.

- 1873-The idea that one needed a constant, now known as Newton's gravitational constant to obtain the gravity force. $F=f \frac{M m}{r^{2}}$ was mentioned explicitly by Cornu, and Baille [5], with notation $f$ for the gravity constant. It was needed because of the practice of defining mass in terms of the $\mathrm{kg}$. The gravitational constant was then used to remove an element from an arbitrary mass that had nothing to do with gravity and include what had been missing in the kg definition of mass, namely the Planck length. This inclusion appears to have occurred subtly, without physicists actually examining or challenging this evolution in the conception of mass, see [6].

- 1894-The gravitational constant was first called $G$ by Boys in August 1884, see [7]. Here he stated the following formula: Force $=G \frac{\text { Mass } \times \text { Mass }}{\text { Distance }^{2}}$, although this latest step going from $f$ to $G$ is merely cosmetic. Even in the early 1900s, many physicists still used $f$. For example, see Isaachsen [8] who had studied under Helmholtz. Max Planck also uses the notation $f$ for the gravity constant in 1899, 1906, and even as late as 1928 [9] [10] [11]. Einstein [12] uses $k$ for the gravitational constant in 1911 and 1916 . However, by the 
1920 s, today's standard notation of $G$ had taken hold more completely.

- 1899/1906 Max Planck [9] [10] derives the Planck units from $G, c$ and $\hbar$ using dimensional analysis: the Planck length $l_{p}=\sqrt{\frac{G \hbar}{c^{3}}}$, the Planck time $t_{p}=\sqrt{\frac{G \hbar}{c^{5}}}$ and the Planck mass $m_{p}=\sqrt{\frac{\hbar c}{G}}$, and the Planck temperature that we will skip here. Until recently it has been believed that one needed to know $G$ to be able to calculate the Planck units. We will challenge that view here.

- 1916-Einstein [13] produces his theory of general relativity, where he "naturally" is heavily dependent on $G$ (he uses notation $K$ ) in his formulas.

- 1916-Einstein [13] suggests that the next big step in the progress of gravity will be to develop a quantum gravity, or in his own words "Because of the intra-atomic movement of electrons, the atom must radiate not only electromagnetic but also gravitational energy, if only in minute amounts. Since, in reality, this cannot be the case in nature, then it appears that the quantum theory must modify not only Maxwell's electrodynamics but also the new theory of gravitation." Einstein

- 1918-Eddington [14] suggests that quantum gravity must be linked to the Planck length, or in his own words: "But it is evident that this length (the Planck length) must be the key to some essential structure. It may not be an unattainable hope that someday a clearer knowledge of the process of gravitation may be reached." However, this contention was ridiculed by other prominent physicists like Bridgman [15] (who received the 1946 Nobel Prize in Physics).

- Recent time-Many researchers [16]-[21], particularly those working on quantum gravity, are more or less convinced that the Planck scale is the smallest possible scale in terms of space and time, and that it is essential for unifying gravity with quantum mechanics. Others have been questioning this view because it seems like we only can find the Planck units through dimensional analysis based on the knowledge of $G, \hbar$, and $c$. See for example [22]. If we not can detect the Planck scale and only calculate the Planck units from dimensional analysis, why not simply abandon the hypothesis of their existence? Our findings in this paper show that the Planck scale is real and indirectly easily detectable from gravity phenomena.

- 2019/2021-For more than 100 years, physicists have tried to unify theories of gravity and quantum mechanics with minimal success and little progress. The recently published theory of collision space-time [6] [23] [24] offers a fresh, new perspective that seems to unify a modified relativity theory with gravity and quantum mechanics in a fully consistent way. This process should not be taken for granted, and further careful study of this theory is warranted. In the end, discussions and investigations by many researchers over time will be the best way to evaluate its merits. The Planck length and 
Planck time are the very essences of that theory.

The importance of further understanding the Planck time is expressed by, for example, Ball, for example, [25] in 1999 where he states: "A physics to match the Planck timescale is the biggest challenge to physicists in the coming century." We will show that the Planck time can be measured from a pendulum clock (grandfather clock) with no knowledge of $G$.

It is important to understand that Newton never invented a gravitational constant, nor did he use one himself. Still, from his theories and insights, he was able to calculate the relative masses of heavenly objects, such as the Earth, the Sun, Jupiter, and Saturn; see the Principia and [3], for example. He was also able to calculate their approximate relative densities. Newton claimed and showed that the weight was proportional to mass. Mass for Newton was a quantity of matter, and he thought matter consisted of indivisible particles. Weight was not linked to $\mathrm{kg}$ in those days because the kilogram definition of mass was first was put forward in 1796, long after Newton's death.

At a deeper level, The $\mathrm{kg}$ is a collision frequency ratio, as shown by Haug [6] [26], and the rest mass in $\mathrm{kg}$ for any mass (from the subatomic particle to super massive galaxies, and even the mass of the observable universe) can be described as

$$
m=\frac{\hbar}{\bar{\lambda}} \frac{1}{c}
$$

where $\hbar$ is the Planck constant, $\bar{\lambda}$ is the reduced Compton wavelength [27] of the mass in question, and $c$ is the speed of light. This calculation is simply the Compton wavelength formula solved for $m$. We do not need to know $m$ to find the Compton wavelength first. Some people will likely question why we are not using the de Broglie [28] [29] matter wavelength, as it is normally the wavelength linked to matter. We think one of the greatest mistakes in physics has been to build quantum and matter theory around the de Broglie wavelength rather than the Compton wavelength. For example, the de Broglie wavelength, $\lambda=\frac{h}{m v \gamma}$, is not mathematically defined for rest-mass particles because we cannot divide by zero ( $v=0$ ), or alternatively, we [30] can claim it converges to infinity as the particle almost comes to rest-something that is absurd. However, the de Broglie wavelength has been accepted as the matter wavelength without hardly anyone questioning it, partly because it has been in use in theoretical physics for almost 100 years. It was assumed in 1927 by the Davisson-Germer [31] experiment for electrons and the experiment by Thomson and Reid [32] that the de Broglie hypothesis was confirmed. This situation is not the case in our view. These experiments only confirm that matter also had wave-like properties, but not that this wavelength was equal to the de Broglie wavelength, one could just as well have claimed it confirmed that matter is linked to the Compton wavelength.

One obtains (mostly) the correct predictions when building a theory using the de Broglie wavelength, but at an unnecessarily complex cost. In our view, the de 
Broglie wavelength is a mathematical derivative of the true matter wavelength, which we claim is the Compton wavelength; this hypothesis is discussed in more detail in [6] [23]. The relationship between the Compton wavelength and the de Broglie wavelength is that the de Broglie wavelength is always the Compton wavelength multiplied by $\frac{c}{v}$. Naturally, then the Compton wavelength is equal to the de Brolgie wavelength times $\frac{v}{c}$, but the de Brolgie wavelength is undefined for rest-mass particles, while the Compton wavelength is always well defined.

However, the standard mass definition (even if we write it as a function of the de Broglie wavelength instead) does not contain any information about how long each collision lasts. For this detail, one needs the unknown length of the indivisible particle $x$, which we have already shown is the Planck length in our previous work [6] [24]. Here we will show that it can be extracted from a simple pendulum (clock) with no knowledge of the so-called Newton's gravitational constant if one combines it with an understanding of collision space-time. Gravity is directly linked to collision space-time. Modern physics has indirectly partly incorporated this in gravity by combining the $\mathrm{kg}$ mass definition with the gravitational constant, because we have $G M$ in most predictable observable gravitational phenomena and never GMm. This fact is covered quite thoroughly in [6] [23] [33]. We will concentrate on how our new understanding of mass makes it possible to measure the Planck time with a pendulum clock.

\section{Using a Pendulum Clock to Find the Planck Time}

Haug [6] [34] has shown how to find the Planck length independently of both $G$ and $\hbar$ using a Cavendish apparatus, and without $G$ using a Newton force spring, see [35]. We extend this work to show how a grandfather pendulum clock can be used to find the Planck time (and thereby naturally the Planck length as well) independently of any knowledge of $G$. Huygens [1] was the first to derive the formula for the period of an ideal mathematical pendulum

$$
T=2 \pi \sqrt{\frac{L}{g}}
$$

where $L$ is the length of the pendulum, and $g$ is the gravitational acceleration. The gravitational acceleration at the surface of the Earth is experimentally known to be about $g \approx 9.81 \mathrm{~m} / \mathrm{s}^{2}$. From modern Newtonian gravity, including the gravitational constant $G$, we also know that the gravitational acceleration is given by

$$
g=\frac{G M}{R^{2}}
$$

However, based on our new collision space-time quantum gravity theory, any rest-mass can be viewed as collision-time, which is given by

$$
\bar{m}=\frac{l_{p}}{c} \frac{l_{p}}{\bar{\lambda}}
$$


where $l_{p}$ is the diameter of an indivisible particle and $\bar{\lambda}$ is the reduced Compton wavelength of the mass in question. Be aware that this formula holds for any mass, even large astronomical bodies like the Earth and the Sun. The Earth does not have a physically reduced Compton wavelength, but we predict that each elementary particle making up the Earth has one. These particles are aggregated in the following formula

$$
\bar{\lambda}=\sum_{i=1}^{n} \frac{1}{\frac{1}{\bar{\lambda}_{1}}+\frac{1}{\bar{\lambda}_{2}}+\cdots+\frac{1}{\bar{\lambda}_{n}}}
$$

Be aware that masses are just functions of the Compton wavelength in terms of collision-time. For example, the standard addition rule of masses is still the same as before. The addition rule for Compton wavelength in composite masses is the same whether one uses the kg mass definition or the collision-time mass definition.

Based on this fact, the gravitational acceleration of any object must be

$$
g=\frac{c^{3} \bar{m}}{R^{2}}
$$

Thus, we have simply replaced the standard mass measure with our mass measure, and we have replaced the gravitational constant with $c^{3}$, which is the much simpler "gravity" constant in our reformulated quantum gravity theory-the speed of light cubed. In other words, we are getting rid of a constant, namely $G$. Actually $G, \hbar$ and $c$ is replaced with only $l_{p}$ and $c$. Now we can rewrite Huygens formula and solve it with respect to the mass, this calculation gives:

$$
\begin{gathered}
T=2 \pi \sqrt{\frac{L}{\frac{c^{3} \bar{m}}{R^{2}}}} \\
\frac{T^{2}}{4 \pi^{2}}=\frac{L}{\frac{c^{3} \bar{m}}{R^{2}}} \\
\bar{m}=\frac{4 \pi^{2} R^{2} L}{c^{3} T^{2}}
\end{gathered}
$$

Next keep in mind that we claim $\bar{m}=\frac{l_{p}}{c} \frac{l_{p}}{\bar{\lambda}} ;$ this proposal means we can solve with respect to $l_{p}$ and this gives

$$
\begin{aligned}
& \frac{l_{p}^{2}}{c \bar{\lambda}}=\frac{4 \pi^{2} R^{2} L}{c^{3} T^{2}} \\
& l_{p}=\frac{2 \pi R \sqrt{L \bar{\lambda}}}{c T}
\end{aligned}
$$

We can divide this by $c$ and get the Planck time

$$
t_{p}=\frac{2 \pi R \sqrt{L \bar{\lambda}}}{c^{2} T}
$$


However, some people may claim that we cannot know the Compton wavelength of the Earth $\bar{\lambda}$ without knowing $G$. This situation is not the case. We can measure the Compton wavelength of an electron from Compton [27] scattering, $\lambda_{e}=\frac{\lambda_{1}-\lambda_{2}}{1-\cos \theta}$. That is, we need to shoot photons at an electron and measure the wavelength (frequency) of the photon before, $\lambda_{1}$, and after, $\lambda_{2}$, the impact with the electron. In addition, we need to measure the angle between the incoming and outgoing photons $(\theta)$. Alternatively, we could use hydrogen spectrum lines to extract the Compton wavelength of the electron; see the Appendix. The cyclotron frequency is linearly proportional to the reduced Compton frequency. One can find the reduced Compton frequency ratio between the proton and the electron by conducting a cyclotron experiment. For example, [36] measured it to be about (see also [37])

$$
\frac{\frac{c}{\bar{\lambda}_{P}}}{\frac{c}{\bar{\lambda}_{e}}}=\frac{f_{P}}{f_{e}}=\frac{\bar{\lambda}_{e}}{\bar{\lambda}_{P}}=1836.152470(76)
$$

They measured the proton-electron mass ratio this way and not the mass in $\mathrm{kg}$. To measure the relative mass between particles rather than their mass in $\mathrm{kg}$ is very similar (in spirit) to Newton measuring the relative mass between planets again without having a $\mathrm{kg}$ or a similar mass measure involved.

This calculation means the Compton frequency of a proton is approximately 1836 times higher than it is in an electron. We now have to count the number of protons on the Earth. This number is theoretically possible, even if not practical (without going an indirect route). Assume we have counted the number of protons (we assume neutrons are the same for simplicity) in the Earth and found that there are approximate $3.57 \times 10^{51}$ protons in the Earth. The Compton frequency (internal collision frequency) in the Earth must then be $3.57 \times 10^{51} \times \frac{c}{\lambda_{e}} \times 1836 \approx 5.089 \times 10^{75}$ times per second. This conclusion means the Compton wavelength of the Earth is $\bar{\lambda} \approx \frac{c}{5.089 \times 10^{75}} \approx 5.89 \times 10^{-68} \mathrm{~m}$. Please take note that we found this number without relying on the Planck constant. Therefore, we have avoided the $\mathrm{kg}$ definition of mass. The speed of light is known - the pendulum time on a $25 \mathrm{~cm}$ pendulum we measure to be about 1 second. We can now input this variable into our formula. If the formula is based on sound logic, it should give an accurate value of the Planck time

$$
t_{p}=\frac{2 \pi \times 6371000 \sqrt{0.25 \times \bar{\lambda}}}{c^{2} \times 1} \approx 5.4 \times 10^{-44} \mathrm{~s}
$$

which is the well-known Planck time. In other words, we have mathematically proven that a pendulum clock can be used to find the smallest time unit without any knowledge of $G$ or the Planck constant. Of course, we could never have counted the number of protons in the Earth in practice. However, simple indi- 
rect ways to do so exist. We could use a Cavendish apparatus to measure the Schwarzschild radius of a lead ball. No knowledge of $G$ or the Planck constant is required to do this calculation. The formula is given by

$$
R_{s}=\frac{L 8 \pi^{2} R^{2} \theta}{c^{2} T^{2}}
$$

This number is the Schwarzschild radius from the large lead ball in a Cavendish apparatus, where $R$ is the distance between the large and the small ball in the apparatus. Again $G$ or $\hbar$ is not needed. Now we can count the number of protons in the large lead ball. This number also represents a practical challenge, but it is theoretically and even practically possible. For example, one has recently been able to count the number of atoms in ${ }^{28} \mathrm{Si}$ crystal spheres, see [38] [39]. Alternatively, we can use the Planck constant. Assume our large lead ball is half a $\mathrm{kg}$; the Compton frequency in half a $\mathrm{kg}$ is given by $f=\frac{c}{\frac{\hbar}{m c}}=\frac{m c^{2}}{\hbar}$. And the Schwarzschild radius of the Earth can be found by the following formula

$$
R_{s, E}=2 g \frac{R^{2}}{c^{2}} \approx 0.0089 \mathrm{~m}
$$

The Compton frequency in the Earth is now the Compton frequency we found in the lead ball multiplied by the Schwarzschild radius of the Earth divided by the Schwarzschild radius of the ball. So, we can easily find the Planck time and Planck length without relying on $G$, or even $\hbar$ if we count the number of protons with a method that does not rely on the Planck constant.

It is impressive that we can indirectly use a grandfather pendulum clock to measure the smallest time interval. How can this be? The pendulum clock is a type of gravity clock; the pendulum periodicity depends on gravitational acceleration. What we have extracted are the shortest possible collision time and shortest possible length (collision length). If we want to know the aggregated collision time, we do not need to know the Compton frequency of the Earth because this number only is used to divide the total collision time into its building blocks-the Planck time duration collisions. In other words, we evaluate the collision time of the entire Earth (per shortest possible time interval), which is given by

$$
\frac{1}{2} T_{s}=\frac{R_{s}}{2 c}=\frac{4 \pi^{2} R^{2} L}{c^{3} T^{2}}
$$

where $T_{s}$ is what we will call Schwarzschild time, $R$ is the radius of the Earth, $T$ is the measured pendulum oscillation time, $L$ is the length of the pendulum, and $c$ is the speed of light. This calculation (the collision time of the Earth) is half the Schwarzschild radius divided by the speed of light.

But why can we measure the Schwarzschild radius or the Schwarzschild time of the Earth using only a grandfather pendulum clock? Is the Schwarzschild radius not related to black holes, where all the mass is pulled inside a black hole within the Schwarzschild radius that only is approximately $9 \mathrm{~mm}$ for the Earth? 
We question this view that the Schwarzschild radius is related to black holes. We think half the Schwarzschild radius is the collision length of indivisible particles making up the gravity object. This indivisible particle is incredibly small, but it is the most important element-the essential building block of energy and mass.

\section{Earth Moon as Giant Great Grandfather Clock (Pendulum Clock) Can Be Used to Find the Planck Length}

The Huygens pendulum clock formula is, in general, only a good approximation for a small angle. It is an inaccurate approximation when the pendulum angle is large, but it is remarkably exact when the Pendulum angle is 360 degrees, that is, a full circle [40]. We can look at the Moon and Earth system as a giant pendulum clock where the length of the pendulum is the distance from the Earth to the Moon. We can find the Planck time from this pendulum clock with the following formula

$$
t_{p}=\frac{2 \pi \sqrt{R^{3} \bar{\lambda}_{E}}}{c^{2} T}
$$

this formula is the same as 9 , except we here also can set $L=R$. Further, $\bar{\lambda}_{M}$ is the reduced Compton wavelength of the large mass (in this case, the Earth), and $R$ is the distance between the Moon and the Earth (length of the pendulum). The distance Earth to the Moon is approximately 384,400,000 meters, the time for the Moon to go around the Earth is approximately 27.32 days (sidereal month), and the reduced Compton wavelength of the Earth is approximate $5.89 \times 10^{-68}$ meter, we now can plug this into the formula above, this gives

$$
t_{p}=\frac{2 \pi \sqrt{384400000^{3} \times 5.89 \times 10^{-68}}}{299792458^{2} \times 27.32 \times 24 \times 60 \times 60} \approx 5.4 \times 10^{-44} \mathrm{~s}
$$

That is, we have a very accurate pendulum clock. The challenge is naturally to find the reduced Compton wavelength of the Earth, but that can be done as described in previous sections of this paper. The Moon to earth distance can be found by parallax, the time for the Moon to go around the Earth we can find (at least approximately) by simply counting the number of days between two full moons. The speed of light we can measure or lookup. We have all the inputs, and the gigantic pendulum clock of the Earth Moon system gives us the Planck time. We could alternatively have used the Sun and Earth system, the pendulum length would then be the distance between the Earth and the Sun, and the relevant Compton wavelength would be that of the Sun, and the time input is how long it takes for the Earth to travel around the Sun. Formula 16 holds for any small mass, moon circulating a planet, or any planet circulating a sun (star), as long as the path is close to circular. In practice, there are natural disturbances since there often are many planets in a solar system, etc. "pulling" on each other.

The Schwarzschild time of the Earth (gravitational mass), which is simply the Schwarzschild radius of the Earth divided by the speed of light, is also given by 


$$
T_{s}=\frac{R_{s}}{c}=\frac{8 \pi^{2} R^{3}}{T^{2} c^{3}} \approx 2.99 \times 10^{-11} \mathrm{~s}
$$

where $R$ is the distance from the Earth to the Moon (pendulum length). The Schwarzschild time of the Earth can also be found by simply $T_{s}=\frac{R_{s}}{c}=\frac{2 G M}{c^{3}}$, but using this method we need to know $G$, which is not needed in the method above.

The Planck time is related to the Schwarzschild time by the following formula

$$
t_{p}=\sqrt{\frac{T_{s} \bar{\lambda}}{2 c}} \approx \sqrt{\frac{2.99 \times 10^{-11} \times 5.89 \times 10^{-68}}{2 c}} \approx 5.4 \times 10^{-44} \mathrm{~s}
$$

In Haug's unified quantum gravity theory, half the Schwarzschild time is the collision-time in any mass per Planck time, see [6].

\section{Conclusions}

We have shown how a simple grandfather clock (a pendulum clock) can be used to measure the shortest time interval there is, namely the Planck time. This calculation can be done with no knowledge of $G$ or the Planck constant. Without the Planck constant, it is difficult at a practical level but fully possible in principle, albeit it would be a very expensive experiment. If we take advantage of the Planck constant, we can easily do it in practice. This result shows that the so-called Newton gravitational constant is not essential for finding the Planck units. The Planck constant is not needed for gravity if one redefines mass in a model that considers what is likely to be the most fundamental form of mass, namely, an indivisible particle.

It is also worth mentioning that Newton never invented or used a gravitational constant. We claim that Newton's original theory is, in many ways, superior to today's modified version of his formula. The ancient formula made one wonder what mass truly was, and we now assume we know what mass is. The mass should be relative to the Planck mass that, in reality, likely is a collision between two indivisible particles. To relate the mass to an arbitrary quantity like the $\mathrm{kg}$ and, in addition, failing to grasp mass at a deeper level truly has caused much confusion and constrained progress and understanding in physics, or as Jammer [41] has pointed out, "Mass is a mess". As suggested by Newton himself, mass is directly linked to an indivisible particle, which we claim has a diameter equal to the Planck length. This paper is one of a series of papers strongly supporting this view.

In conclusion, we assert that in principle, we cannot measure the smallest possible time with an iPhone, an atomic clock, or even the most advanced optical clock, but rather with an old-fashioned grandfather pendulum clock. The key difference is that the grandfather clock is a gravity clock, and the shortest time interval is directly linked to the building blocks of mass, which are linked to gravity. 


\section{Acknowledgements}

Thanks to Victoria Terces for editing and Stephan Wo for useful comments.

\section{Conflicts of Interest}

The author declares no conflicts of interest regarding the publication of this paper.

\section{References}

[1] Huygens, C. (1673) Horologium oscillatorium sive de motu pendularium.

[2] Newton, I. (1686) Philosophiae Naturalis Principia Mathematica. Joseph Streater, London. https://doi.org/10.5479/sil.52126.39088015628399

[3] Cohen, I.B. (1998) Newton's Determination of the Masses and Densities of the Sun, Jupiter, Saturn, and the Earth. Archive for History of Exact Sciences, 53, 83-95. https://doi.org/10.1007/s004070050022

[4] Cavendish, H. (1798) Experiments to Determine the Density of the Earth. Philosophical Transactions of the Royal Society of London, (Part II), 88, 469-526. https://doi.org/10.1098/rstl.1798.0022

[5] Cornu, A. and Baille, J.B. (1873) Détermination nouvelle de la constante de l'attraction et de la densité moyenne de la terre. Comptes rendus de 1 Académie des Sciences Paris, 76, 1.

[6] Haug, E.G. (2020) Collision-Space-Time: Unified Quantum Gravity. Physics Essays, 33, 46-78. https://doi.org/10.4006/0836-1398-33.1.46

[7] Boys, C.V. (1894) The Newtonian Constant of Gravitation. Nature, 50, 571. https://doi.org/10.1038/050571a0

[8] Isaachsen, D. (1905) Lærebog i Fysikk. Det Norske Aktieforlag.

[9] Planck, M. (1899) Natuerliche Masseinheiten. Der Königlich Preussischen Akademie Der Wissenschaften.

[10] Planck, M. (1906) Vorlesungen über die Theorie der Wärmestrahlung. J.A. Barth, Leipzig, 163. The English Translation “The Theory of Radiation” (1959) Dover.

[11] Planck, M. (1928) Einführung in die allgemeine Mechanik. Verlag von Hirtzel, Leipzig.

[12] Einstein, A. (1911) Über den einfluss der schwercraft auf die ausbreitung des lichtes. Annalen der Physik, 35, 898-908. https://doi.org/10.1002/andp.19113401005

[13] Einstein, A. (1916) Näherungsweise integration der feldgleichungen der gravitation. Sitzungsberichte der Königlich Preussischen Akademie der Wissenschaften Berlin.

[14] Eddington, A.S. (1918) Report on the Relativity Theory of Gravitation. The Physical Society of London, Fleetway Press, London.

[15] Bridgman, P.W. (1931) Dimensional Analysis. Yale University Press, New Haven.

[16] Padmanabhan, T. (1985) Planck Length as the Lower Bound to All Physical Length Scales. General Relativity and Gravitation, 17, 215-221. https://doi.org/10.1007/BF00760244

[17] Padmanabhan, T. (1985) Physical Significance of Planck Length. Annals of Physics, 165, 38-58. https://doi.org/10.1016/S0003-4916(85)80004-X

[18] Adler, R.J. (2010) Six Easy Roads to the Planck Scale. American Journal of Physics, 78, 925-932. https://doi.org/10.1119/1.3439650 
[19] Hossenfelder, S. (2012) Can We Measure Structures to a Precision Better than the Planck Length? Classical and Quantum Gravity, 29, Article ID: 115011. https://doi.org/10.1088/0264-9381/29/11/115011

[20] Hossenfelder, S. (2013) Minimal Length Scale Scenarios for Quantum Gravity. Living Reviews in Relativity, 16, Article No. 2. https://doi.org/10.12942/lrr-2013-2

[21] Faraoni, V. (2017) Three New Roads to the Planck Scale. American Journal of Physics, 85, 865-869. https://doi.org/10.1119/1.4994804

[22] Unzicker, A. (2020) The Mathematical Reality: Why Space and Time Are an Illusion. Independently.

[23] Haug, E.G. (2020) Rethinking the Foundation of Physics and Its Relation to Quantum Gravity and Quantum Probabilities: Unification of Gravity and Quantum Mechanics. https://vixra.org/abs/2012.0089

[24] Haug, E.G. (1921) Quantum Gravity Hidden in Newton Gravity and How to Unify it with Quantum Mechanics. In: The Origin of Gravity from the First Principles, NOVA Publishing, New York, 135-218.

[25] Ball, P. (1999) Physics at the Planck Time. Nature, 402, C61. https://doi.org/10.1038/35011550

[26] Haug, E.G. (2018) Newton and Einstein's Gravity in a New Perspective for Planck Masses and Smaller Sized Objects. International Journal of Astronomy and Astrophysics, 8, 6-23. https://doi.org/10.4236/ijaa.2018.81002

[27] Compton, A.H. (1923) A Quantum Theory of the Scattering of X-Rays by Light Elements. Physical Review, 21, 483-502. https://doi.org/10.1103/PhysRev.21.483

[28] Broglie, L. (1923) Waves and Quanta. Nature, 112, 540. https://doi.org/10.1038/112540a0

[29] Broglie, L. (1924) Recherches sur la théorie des quanta. PhD Thesis (Paris).

[30] Lvovsky, A.I. (2018) Quantum Physics: An Introduction Based on Photons. Springer, Berlin.

[31] Davisson, C. and Germer, L.H. (1927) Diffraction of Electrons by a Crystal of Nickel. Physical Review, 30, 705-741. https://doi.org/10.1103/PhysRev.30.705

[32] Thomson, G.P. and Reid, A. (1927) Diffraction of Cathode Rays by a Thin Film. Nature, 119, 890. https://doi.org/10.1038/119890a0

[33] Haug, E.G. (2021) Demonstration That Newtonian Gravity Moves at the Speed of Light and Not Instantaneously (Infinite Speed) as Thought! Journal of Physics Communication, 5, Article ID: 025005. https://doi.org/10.1088/2399-6528/abe4c8

[34] Haug, E.G. (2017) Can the Planck Length Be Found Independent of Big G? Applied Physics Research, 9, 58-66. https://doi.org/10.5539/apr.v9n6p58

[35] Haug, E.G. (2020) Finding the Planck Length Multiplied by the Speed of Light without Any Knowledge of $G$, $c$, or $h$, Using a Newton Force Spring. Journal Physics Communication, 4, Article ID: 075001. https://doi.org/10.1088/2399-6528/ab9dd7

[36] Van-Dyck, R.S., Moore, F.L., Farnham, D.L. and Schwinberg, P.B. (1985) New Measurement of the Proton-Electron Mass Ratio. International Journal of Mass Spectrometry and Ion Processes, 66, 327-337. https://doi.org/10.1016/0168-1176(85)80006-9

[37] Gräff, G., Kalinowsky, H. and Traut, J. (1980) A Direct Determination of the Proton Electron Mass Ratio. Zeitschrift für Physik A Atoms and Nuclei, 297, 35-39. https://doi.org/10.1007/BF01414243

[38] Becker, P. (2012) The New Kilogram Definition Based on Counting the Atoms in a 
${ }^{28}$ Si Crystal. Contemporary Physics, 53, 461-479.

https://doi.org/10.1080/00107514.2012.746054

[39] Bartl, G., et al. (2017) A New ${ }^{28}$ Si Single Crystal: Counting the Atoms for the New Kilogram Definition. Metrologia, 54, 693-715.

[40] Haug, E.G. (2020) The Huygens Formula Is Exact for a Very Large Angle: The Moon-Earth System as a Gigantic Pendulum Clock. Working Paper Norwegian University of Life Sciences, Ås.

[41] Jammer, M. (2000) Concepts of Mass in Contemporary Physics and Philosophy. Princeton University Press, Princeton. https://doi.org/10.1515/9781400823789

[42] Haug, G.E. (2020) The Two Relativistic Rydberg Formulas of Suto and Haug: Further Comments. Journal of Modern Physics, 11, 1938-1949.

https://doi.org/10.4236/jmp.2020.1112122

\section{Appendix: Finding the Compton Wavelength of the Electron}

There are several ways to find the Compton wavelength of the electron. One way is to use Compton scattering. The Compton scattering method has the advantage that it needs no knowledge of the Planck constant to find the Compton wave. Another method to find the Compton wavelength of the electron is to watch the hydrogen spectral lines. The Compton wavelength of the electron is linked to the spectral lines (of Hydrogen atoms) with the following formula [42]

$$
\lambda_{e}=\lambda\left(\frac{1}{\sqrt{1-\alpha^{2} / n_{1}^{2}}}-\frac{1}{\sqrt{1-\alpha^{2} / n_{2}^{2}}}\right)
$$

This method also requires that we know the fine structure constant, which means that we also need to know the Planck constant. For hydrogen-like atoms with elements above $z>1$, the following formula can be used to find the Compton wavelength

$$
\lambda_{e}=\lambda\left(\frac{1}{\sqrt{1-\left(z \alpha / n_{1}\right)^{2}}}-\frac{1}{\sqrt{1-\left(z \alpha / n_{2}\right)^{2}}}\right)
$$

